\title{
Los efectos de la luna en la producción agropecuaria
}

Aura Danelia Vásquez Cabrera ${ }^{5}$ Jaritza Maquiel Narváez Gago ${ }^{6}$ Wilson Antonio Calero Borge ${ }^{7}$

\section{Resumen}

T a investigación se realizó en la colonia San Martín, ubicada a 30 km al Noreste de Nueva Guinea L(RAAS). El propósito fue caracterizar el conocimiento tradicional que tienen los productores acerca del efecto de la luna en la producción agropecuaria. Se apoyó en la aplicación de entrevistas dirigida a productores y productoras. Los participantes fueron seleccionados por la actividad productiva a que se dedican, residentes en la colonia San Martín, así como las disponibilidad a participar en el estudio, igualmente se aplicó entrevistas a productoras jefas de familia. Los productores consideran de importancia las fases lunares en la producción agropecuaria, ya que estas influyen directamente en sus cosechas y es una guía para aprovechar mejor la tierra y sus cultivos. El período más adecuado para casi todas las actividades pecuarias y agrícolas es luna llena. Ellos conocen de las fases lunares y efectos diversos en los cultivos y animales, los que practican día a día y fueron heredados por sus antepasados. La mujer se involucra en las distintas actividades del campo; la mujer está pendiente de las fases lunares adecuadas para la realización de actividades productivas y cosecha de sus productos. Valoran muy eficaz y positivo trabajar de acuerdo al calendario lunar, ya que obtienen beneficios, y por ende estos conocimientos tradicionales los heredaran a las futuras generaciones.

Palabras clave: conocimiento tradicional; cosechas.

\section{Introducción}

El conocimiento tradicional que tiene el productor sobre los efectos de la luna en la producción agrícola y pecuaria son prácticas muy antiguas que han adquirido de sus ancestros y que aplican en la actualidad, constituyendo así un enfoque significativo en el uso adecuado de sus tradiciones obteniendo excelentes beneficios (Franco, 2010).

El Municipio de Nueva Guinea es agropecuario, por lo que los conocimientos acerca de la producción son de suma importancia. Los conocimientos que tienen los agricultores los induce a confiar plenamente en las prácticas agrícolas que realizan, lo cual significa que consideran que la luna tiene influencia en la producción razón por la cual deben trabajar de acuerdo al calendario lunar, conocimientos tradicionales que heredarán a las futuras generaciones.

\footnotetext{
5 Licdas. en Ciencias de la Educación con mención enBiología. E-mail: investpost.ng@uraccan.edu.ni

6 Licdas. en Ciencias de la Educación con mención enBiología. E-mail: investpost.ng@uraccan.edu.ni

7 Master en Ciencias en Agroforestería. Coordinador en Invest. y posgrado, URACCAN-Nueva Guinea. E-mail: investigacion.ng@uraccan.edu.ni
} 
Diversos estudios abordan la importancia que le conceden los productores a las fases lunares para realizar la siembra y cosecha de sus cultivos, llegando incluso a regir su calendario agrícola según las fases lunares, en este sentido es importante documentar este conocimiento para que pueda ser socializado con los productores.

La presente investigación se realizó para caracterizar los conocimientos tradicionales que tienen los productores y productoras acerca del efecto de las fases lunares en la actividad productiva y brindar alternativas para un uso adecuado en los sistemas productivos y obtener mejores rendimientos. Con esta investigación se aporta a la sistematización de conocimientos tradicionales de los productores y cómo los mismos se transmiten de generación en generación.

\section{Marco teórico}

\section{Conocimientos tradicionales}

Los conocimientos tradicionales son todas aquellas sabidurías ancestrales y conocimientos colectivos e integrales que poseen los pueblos indígenas, afroamericanos y comunidades locales fundamentadas en la praxis milenaria y su proceso de interacción hombre-naturaleza, y transmitidos de generación en generación, habitualmente de manera oral (CAN, 2005).

\section{Importancia del calendario lunar}

La luna marca muchas de nuestras actividades como seres humanos, los días y las noches, son un reflejo de su importancia en nuestras vidas, retomando a Alvarenga (1996), se resumen algunos elementos de la importancia del calendario lunar:

- A los biólogos y otros recolectores de muestras naturales les sirve para planificar la observación, documentación y recolección de insectos, animales y otros materiales que se ven afectados por la cantidad de luz nocturna.

- A los observadores de la Luna y del cielo nocturno, les sirve para planificar las horas y días de observación y dimensionar sus expectativas.
- Todos los que estudian y explotan la flora y la fauna de la zona intermareal. (Cubierta en marea alta y descubierta en marea baja) requieren del conocimiento de las mareas y las fases lunares.

- Los agricultores se guían por las fases lunares en la mayoría de los procesos de manejo de cultivos.

- En la ganadería, algunos se rigen por las fases lunares para planificar la inseminación artificial y otros sistemas de propagación de especies.

\section{Influencia de la luminosidad lunar}

Según Restrepo (2005), la luminosidad lunar es esencial para el desarrollo de la vida de las plantas, a diferencia de la luz solar, la luz lunar ejerce directamente una fuerte influencia sobre la germinación de las semillas, ya que sus rayos penetran con relativa profundidad contrario a lo que sucede con los rayos solares que no penetran en su intimidad. De igual manera está demostrado que la actividad de la fotosíntesis es superior en todas las plantas a partir de la luna creciente hacia la luna llena donde se da el mayor incremento del proceso fotosintético en las plantas, fenómeno atribuido científicamente al incremento de la luz lunar sobre la tierra.

\section{Influencia de las fases lunares en el mo- vimiento de la savia en las plantas}

Las actividades de la poda de los duraznos, los perales, las manzanas, el arreglo de las parras y el corte de los árboles maderables está limitado casi exclusivamente a los cuatro meses del año, como son mayo, junio, julio y agosto a la vez, estas actividades limitan a las diferentes fases lunares, pues de lo contrario las podas y los cortes de madera fuera de estas épocas, arrojarían resultados nada gratificantes para los campesinos, como frutales débiles, con poca producción de frutos y pequeños, las maderas más livianas, predispuestas a rajarse y convertirse en un atractivo plato para los comejenes (Restrepo, 2005).

\section{La luminosidad lunar y su influencia con la lluvia}

Entre la luna y el agua que hay sobre la Tierra existe cierto tipo de relación. Supongamos que acaban de transcurrir unos días de lluvia y que a estos días de lluvia les sucede la luna llena. Con las 
fuerzas que vienen de la luna en los días del plenilunio ciertamente ocurre algo colosal sobre la Tierra: estas fuerzas se introducen en toda la vida vegetal (no podría hacerlo si no antecedieran los días de lluvia; Steyner, 1924).

Por lo tanto, deberemos hablar de la importancia que sembremos semillas cuando han caído lluvias en cierta forma y luego viene el brillo de la luna llena, o si se puede sembrar en cualquier momento, sin tener en cuenta nada en especial la acción de la luna llena es impetuosa y potente en ciertas plantas después de días de lluvia, débil y escasa tras días en que ha brillado el Sol estas cosas estaban contenidas en los antiguos refranes campesinos (Colmenares, S/F).

\section{Influencia de la luna en el cultivo de tubérculos}

Si los tubérculos y raíces se cosechan para almacenar y para producir semillas, la fase lunar que les interesa va desde cuarto creciente a la luna llena, porque su cosecha contienen menos agua y hay mucho menos riesgo de que se pudra; por otro lado, algunos prefieren cosechar para la producción de semillas en plena luna menguante y luna nueva, porque en esta etapa se concentran mayor cantidad de nutrientes; otros tienen una opinión que se puede decir intermedia, porque tienen en cuenta también el tiempo que ha de permanecer almacenada su cosecha a la espera de ser llevada de nuevo al campo para plantarse (Miranda, 2009).

Si el tiempo de almacenamiento no es muy largo, entonces cosechan entre la menguante y luna nueva, porque los tubérculos y las raíces contienen más agua; pero si tienen que esperar mucho tiempo, la mejor fase lunar es la que va tres días después de la creciente, hacia la luna llena (tubérculos con menos agua, equivalentes al período de siete días intensivos de aguas arriba) (Colmenares, s.f)

\section{Metodología y materiales}

La investigación se realizó en la colonia San Martín del Municipio de Nueva Guinea (RAAS), dicha comunidad se encuentra al noreste del municipio, a una distancia de, $30 \mathrm{~km}$ con una población de 339 habitantes, que se aglutinan en 90 familias ,210 mujeres, 129 hombres.

Esta investigación es de carácter cualitativo, exploratorio y transversal, por ser este un tema poco estudiado y permitió recabar información relevante. El universo del estudio fueron todas las 90 familias de colonia San Martín, para seleccionar a los participantes en el estudio se aplicaron criterios de selección.

Las técnicas utilizadas fueron a través de entrevista a los productores sobre las prácticas ancestrales en la producción agropecuaria de acuerdo al ciclo lunar, y los instrumentos utilizados fueron los cuestionarios, listado de productores, grabadoras, entre otros.

\section{Resultados y discusión}

\section{Los efectos de las fases de la luna en la producción}

La mayoría de los productores entrevistados coinciden cuando afirman que primeramente hay que conocer e identificar las fases de la luna para poder obtener una mejor producción. De acuerdo a Franco (2010), las respuestas de los productores se fundamentan debido a que existen tradiciones fuertemente arraigadas basadas en el saber tradicional asociadas con épocas, horas de cosecha y períodos de maduración.

Alvarenga (1996) y Arce (1998), dan una pequeña explicación de estas creencias ya que cuando los tres astros están totalmente alineados se producen los eclipses solares es por eso que durante los ciclos lunares, los cambios de la fases de la luna, la cantidad de luz reflejada a la tierra va cambiando la fuerza de gravedad que la luna ejerce sobre el planeta y las plantas se desarrollan de forma balanceada.

Los productores plantean que cuando la cosecha es para el consumo inmediato la fase más adecuada es la de novilunio, porque allí es cuando los frutos son más jugosos, en cambio si es para semilla la mejor fase es luna llena. Esto es coincidente con Alvarenga (1996), quien plantea que los agricultores creen que efectivamente la luna tiene influencia directa en la producción agrícola, por tanto deben guiarse en sus trabajos de acuerdo a las fases de este satélite.

Entre los efectos aducidos por los productores se pueden mencionar cuando estos realizan siembra de plantas en luna nueva; en este caso habrá problemas porque las plantas no producirían satisfactoriamente y las semillas o material vegetativo se pudren mayoritariamente en esta fase lunar. 
Arce (1998), expresa ejemplos relacionados con las respuestas de los productores, que las antiguas civilizaciones realizaban sus prácticas agrícolas acorde con los ritos lunares basados en las creencias de que existen ritmos en los procesos metabólicos de plantas y animales.

Por tal razón es que ellos toman en cuenta los cambios lunares para evitar pudriciones y pérdidas en sus cultivos, ya que los productores afirman que los cambios lunares afectan los movimientos del agua en el suelo. Estas fuerzas que ocurren sobre la tierra se introducen en toda la vida vegetal (Steyner, 1924), durante el ciclo lunar la cantidad de luz reflejada a la tierra va cambiando, el efecto más notable es las mareas; se cree que la luna va afectando los movimientos del agua en el suelo y solubilidad de nutrientes a las raíces de la planta.

\section{Practicas de produción según el ciclo lunar}

Los productores expresaron que realizan diferentes prácticas tomando en cuenta los efectos de la luna tales como: plantaciones de cacao (Theobroma cacao), platanales (Musa sp), ayote (Cucurbita pepo), maíz (Zea mays), frijoles (Phaseolus vulgaris). Paralelamente mencionaron que no realizan ninguna práctica en fases no adecuadas para evitar daños en sus cultivos, a su criterio el período de luna llena es el más adecuado, porque ahí es cuando los frutos tienen mayor calidad; además los frutos no se dañan, ni los afectan las plagas y hay fructificación máxima.

Cuando el productor habla de la fases no adecuadas, ellos se refieren específicamente en la fase de luna nueva, porque en la mayoría del tiempo en esa fase tiende a estar lloviendo por lo cual sus tradiciones le indican que en ese momento no se debe hacer ninguna practica agropecuaria. Arce (1998), apunta que cuando la luna está creciente hacia la media, los procesos iniciados en la tierra también se desarrollan en la misma línea, pero el crecimiento se vuelve más constructivo y produce un fruto digno.

Los productores plantean que la poda de árboles la realizan en luna llena con el fin que sus árboles sean más frondosos evitando así infecciones y favorecen la cicatrización. En cambio si es para desaparecerlos no toman en cuenta el período adecuado.
Según Restrepo (2005), las podas se limitan a las fases lunares propicias pues de lo contrario fuera de estas épocas arrojarían resultados nada gratificantes, como frutales débiles por la pérdida de savia, poca producción de frutos y pequeños, maderas más livianas, predispuestas a rajarse y convertirse en alimento para las termitas. Cabe señalar que Restrepo sigue expresando que las podas y limpiezas de los árboles enfermos las realizan entre las fases de la luna menguante y la luna nueva ,evitando pudriciones y obteniéndose una rápida y mejor cicatrización.

Con respecto a la castración de animales, expresan que las realizan en luna llena con el objetivo que la herida sea cicatrizada más rápidamente y evitar sangrado profuso en los animales y estos aumenten de tamaño y peso. La incubación de huevos la realizan en cualquier fase lunar, excepto en luna nueva ya que en esa fase tienden a salir o nacer con imperfecciones los pollitos, de esa forma evitan perdidas; en cambio algunos respondieron que no tomaban en cuenta las fases lunares por tal razón es que muchas veces los huevos no eclosionan todos.

En relación a la aparición de plagas y enfermedades de los cultivos y animales, los entrevistados consideran que su aparición está relacionada con las fases lunares porque según cada fase crea ciertas condiciones climatológicas, unas veces hay sequías y en otras el clima está muy lluvioso y las plagas se desarrollan de acuerdo a las condiciones climáticas.

\section{Transmisión de conocimientos ances- trales en la comunidad}

Las productoras y productores coinciden en que han adquirido los conocimientos de los efectos de la luna en la agricultura; de sus ancestros, ellos comentan que esos conocimientos se han trasmitido de generación en generación.

Las respuestas de los productores tienen estrecha relación con su cosmovisión y elementos culturales, ya que ellos expresan que sus abuelos, padres, tíos, amigos siempre han tomado en cuenta las fases lunares para la realización de sus actividades pecuarias y agrícolas; reconociendo esas fases como guía para cualquier trabajo a realizar es por eso que ellos continúan poniendo en práctica esas creencias. 


\section{Participación de la mujer en la transmi- sión del conocimiento tradicional}

La mujer juega un papel importante en el proceso de actividades productivas de patio, las cuales se relacionan con las diferentes fases lunares, ya que la mayoría de productoras realizan diferentes practicas como siembra de ayotes (Cucurbita pepo), chayotes (Sechuem edulis), pipianes (Cucurbita argirosperma), pepinos (Cucumis sativus) melón (Cucumis melo), sandías (Citrulus lanatus) tomando como referencia las fases lunares más adecuadas, con el fin de obtener cosechas de forma satisfactoria.

Es importante resaltar que la mujer, además de realizar diferentes prácticas agrícolas de patio, inculque esos conocimientos valiosos en sus hijos y familiares, por otra parte se involucran no solo en las actividades de patio sino que también las distintas responsabilidades del hogar.

\section{Conclusiones}

- Las productoras y productores tienen un amplio conocimiento de los efectos de la luna en la producción agrícola y pecuaria.

- Los productores le da gran importancia a las fases lunares reconociendo que estas son una guía para cualquier actividad agropecuaria.

- Los agricultores creen que efectivamente la luna tiene influencia directa en la producción agrícola y pecuaría razón por la cual deben trabajar en concordancia con las fases de este satélite.

- El período más adecuado para las actividades pecuarias y agrícolas según sus experiencias es el de luna llena, porque es allí cuando los frutos están en el tiempo duradero y fructífero, las plantaciones crecen y desarrollan como el productor las necesita, las cosechas no se pudren, los animales al ser castrados aumentan de tamaño y peso.

- Los conocimientos empíricos los han adquirido y heredado de sus ancestros y los heredan a sus futuras generaciones en el sistema agrícola y pecuario.

- La mujer juega un papel importante en el proceso productivo de patio, ellas son las que están pendiente de la siembra de ayotes, chayotes, pipianes y otros, siempre retomando las fases lunares.

\section{Bibliografía}

Alvarenga Silvana (1996). Influencia de las fases lunares sobre plantas y animales. dep. Biología ITCR.

Arce P Jorge (1998). La luna y la Agricultura. Ing. Agrónomo. EARTH. Costa Rica.

CAN/CAF (2005). Elementos para la protección sui generis de los conocimientos tradicionales colectivos e integrales desde la perspectiva indígena. Caracas.

Colmenares Ricardo (S/f). Agricultura Biodinámica. Urbanización El Roquedal. Hoyo de Manzanares, Madrid

Franco Julia María (2010). Influencia de luna en cultivos. Recuperado el o8 de Mayo del 2012, de http://wwwbing.com/search.Influencia de la luna en la Agricultura 2010.

Miranda Trejo, Herrera Cabrera, Paredes Sánchez, Delgado Alvarado (2009). Conocimiento tradicional sobre predictores Climáticos en la Agricultura en los llanos de Serdán, pueblo México. Vol. 10, Núm. 2 Universidad de Yucatán (México) PP151-16o.

Restrepo Jairo (2005). La luna y su influencia en la Agricultura. Fundación, Juquira Candiru, Colombia- Brasil-México, pp.1-65.

Steiner. Rudolf (1924). Tratado sobre agricultura biológico-dinámica. Primera conferencia PP 125-130. 